\title{
ON THE STRUCTURE OF SELF-SIMILAR FRACTALS
}

\author{
PERTTI MATTILA*
}

\section{Introduction}

A subset $E$ of the Euclidean $n$-space $R^{n}$ is called self-similar if there are similitudes $S_{1}, \ldots, S_{N}$ of $R^{n}$ such that

$$
E=\bigcup_{i=1}^{N} S_{i} E
$$

and the different parts $S_{i} E$ are "nearly" disjoint; more precisely, if $s$ is the Hausdorff dimension of $E$ then $\mathscr{H}^{s}\left(S_{i} E \cap S_{j} E\right)=0$ for $i \neq j$. Here $\mathscr{H}^{s}$ is the $s$-dimensional Hausdorff measure. By a similitude we mean a map $S: R^{n} \rightarrow R^{n}$ such that for some $r$, $0<r<1,|S x-S y|=r|x-y|$ for all $x, y \in R^{n}$. The term fractal, appearing in the title, is a general name introduced by B. Mandelbrot for sets whose Hausdorff and topological dimensions differ from each other.

In [3] Mandelbrot has studied the connections of self-similar fractals to various physical phenomena. In [2] J. E. Hutchinson showed that to any finite family $\mathscr{S}=\left\{S_{1}, \ldots, S_{N}\right\}$ of similitudes of $R^{n}$ corresponds a unique compact set $K \subset R^{n}$ such that $K=\cup_{i=1}^{N} S_{i} K$. This set will be denoted by $|\mathscr{S}|$. Also several properties of selfsimilar fractals were proved in [2]. One of them was that if $m$ is a positive integer, $0<m<n$, and $\mathscr{S}$ satisfies certain natural separation conditions (which are valid, e.g. if the sets $S_{i} K$ are disjoint), then the intersection of $K=|\mathscr{S}|$ with any $m$-dimensional $C^{1}$ submanifold of $R^{n}$ has $m$-dimensional Hausdorff measure zero. In this paper we prove that under a slightly stronger separation condition, the Hausdorff dimension of such an intersection is always at most $m-\varepsilon$ where $\varepsilon>0$ depends only on $\mathscr{S}$ and not on the submanifold in question. This result has some content only if the Hausdorff dimension of $K, \operatorname{dim} K$, is not less than $m$. We also study the case $s=\operatorname{dim} K \leqq m$, and we show that then there are only two possibilities; either $K$ lies on an $m$-dimensional affine subspace of $R^{n}$ or $\mathscr{H}^{s}(K \cap M)=0$ for every $m$-dimensional $C^{1}$ submanifold $M$ of $R^{n}$.

* Supported in part by NSF grant MCS 77-18723 (02).

doi:10.5186/aasfm.1982.0723 


\section{Preliminaries}

We follow the notation and terminology of Hutchinson [2]. In the whole paper

- $m$ and $n$ are integers, $0<m<n$,

- $\mathscr{S}=\left\{S_{1}, \ldots, S_{N}\right\}$ is a finite family of similitudes of $R^{n}$,

$-K=|\mathscr{S}|$,

- $s=\operatorname{dim} K$, the Hausdorff dimension of $K$,

$-C_{p}(N)=\{1, \ldots, N\}^{p}$ for $p=1,2, \ldots$; we denote $\alpha \prec \beta$ if $\alpha \in C_{p}(N)$, $\beta \in C_{q}(N), \quad p \leqq q$, and $\beta$ is an extension of $\alpha$, that is, $\alpha=\left(i_{1}, \ldots, i_{p}\right)$, $\beta=\left(i_{1}, \ldots, i_{p}, i_{p+1}, \ldots, i_{q}\right)$,

- $S_{\alpha}=S_{i_{1}} \circ \ldots \circ S_{i_{p}}$ for $\alpha=\left(i_{1}, \ldots, i_{p}\right) \in C_{p}(N)$

- $A_{\alpha}=S_{\alpha} A$ for $A \subset R^{n}$,

- $r_{i}$ is the Lipschitz constant of $S_{i}$ for $i=1, \ldots, N$ and $r_{\alpha}=r_{i_{1}} \cdot \ldots \cdot r_{i_{p}}$ that of $S_{\alpha}$ for $\alpha=\left(i_{1}, \ldots, i_{p}\right) \in C_{p}(N)$. We assume $0<r_{1} \leqq \ldots \leqq r_{N}<1$.

Observe that if $B \subset R^{n}$ is bounded, then for $\alpha \in C_{p}(N)$

$$
d\left(B_{\alpha}\right)=r_{\alpha} d(B) \leqq r_{N}^{P} d(B) \rightarrow 0 \quad \text { as } \quad p \rightarrow \infty,
$$

where $d$ denotes diameter.

We say that $\mathscr{S}$ satisfies the open set condition if there is an open bounded set $O \subset R^{n}$ such that $\cup_{i=1}^{N} O_{i} \subset O$ and $O_{i} \cap O_{j}=\emptyset$ for $i \neq j$.

If $\mathscr{S}$ satisfies the open set condition with $O$, then $\bar{O} \supset \bar{O}_{i_{1}} \supset \bar{O}_{i_{1} i_{2}} \supset \ldots, K \subset \bar{O}$, $K_{\alpha} \subset \bar{O}_{\alpha}$ for all $\alpha$, and

$$
K=\bigcap_{p=1}^{\infty} \bigcup_{\alpha \in C_{p}(N)} \bar{O}_{\alpha} .
$$

(See $[2,5.2$ (3) (ii) and 3.1 (3) (viii)].) Moreover, $s$ is uniquely determined by the condition $\sum_{i=1}^{N} r_{i}^{s}=1$, and $0<\mathscr{H}^{s}(K)<\infty,[2,5.1$ (2) and $5.3(1)]$. Observe that neither the sets $K_{i}$ nor $\bar{O}_{i}$ need to be disjoint. But if $K_{i}$ 's are disjoint, then $\mathscr{S}$ always satisfies the open set condition with $O=$ an $\varepsilon$-neighborhood of $K$ for sufficiently small $\varepsilon>0$ $[2,5.2(2)]$.

The lower and upper $s$-dimensional densities of a set $A \subset R^{n}$ at a point $x \in R^{n}$ are defined by

$$
\begin{gathered}
\Theta_{*}^{s}(A, x)=\liminf _{r_{\downarrow} 0} \frac{\mathscr{H}^{s}(A \cap B(x, r))}{\alpha(s) r^{s}}, \\
\Theta^{* s}(A, x)=\limsup _{r_{\downarrow} 0} \frac{\mathscr{H}^{s}(A \cap B(x, r))}{\alpha(s) r^{s}},
\end{gathered}
$$

where $B(x, r)$ is the closed ball with centre $x$ and radius $r$ and $\alpha(s)$ is a positive normalization constant. 


\section{The case $m \leqq s$}

In this section we prove

3.1. Theorem. Suppose that $\mathscr{S}$ satisfies the open set condition with the open bounded sets $O$ and $U, O \subset U$, and that the following separation condition holds: $\neq \emptyset$.

If l is a line in $R^{n}$ such that $\bar{O}_{i} \cap l \neq \emptyset \neq \bar{O}_{j} \cap l$ for some $i \neq j$, then $\left(U \sim \bigcup_{i=1}^{N} \bar{O}_{i}\right) \cap l$

Then there is $\varepsilon>0$ such that for any m-dimensional $C^{1}$ submanifold $M$ of $R^{n}$, $\operatorname{dim}(M \cap K) \leqq m-\varepsilon$.

Proof. We shall show that there is $\varepsilon>0$ such that, given $M$, every point $x \in M$ has a neighborhood $W \subset R^{n}$ such that $\operatorname{dim}\left(M \cap K_{\alpha}\right) \leqq m-\varepsilon$ whenever $\bar{U}_{\alpha} \subset W$. To see that this implies $\operatorname{dim}(M \cap K) \leqq m-\varepsilon$, we choose compact subsets $M_{i}$ of $M$ such that $\bigcup_{i} M_{i}=M$. Each $M_{i}$ can be covered with a finite number of such neighborhoods $W$, and for every $i$ there is $p$ such that each $\bar{U}_{\alpha}, \alpha \in C_{p}(N)$, which meets $M_{i}$ is contained in some such neighborhood $W$. It follows that $\operatorname{dim}\left(M_{i} \cap K\right) \leqq m-\varepsilon$; hence also $\operatorname{dim}(M \cap K) \leqq m-\varepsilon$.

Since the closures $\bar{O}_{i}$ are compact, since the function

$$
l \mapsto \sup \left\{\lambda: B(a, \lambda) \cap l \subset\left(U \sim \bigcup_{i=1}^{N} \bar{O}_{i}\right) \cap l \text { for some } a \in l\right\}
$$

is lower semicontinuous in the space of all lines in $R^{n}$ and since it is positive on the compact set of all lines $l$ for which $\bar{O}_{i} \cap l \neq \emptyset \neq \bar{O}_{j} \cap l$ for some $i \neq j$, it has a positive minimum on this set (cf. [2, 5.4 (1)]). This means that there is $\lambda>0$ such that $\bar{O}_{i} \cap l \neq \emptyset \neq \bar{O}_{j} \cap l$ for some $i \neq j$ implies $B(a, \lambda) \cap l \subset\left(U \sim \bigcup_{i=1}^{N} \bar{O}_{i}\right) \cap l$ for some $a \in l$. Applying the similitudes $S_{\alpha}, \alpha \in C_{p}(N)$, we find that for any line $l$

$$
\begin{gathered}
\bar{O}_{\alpha i} \cap l \neq \emptyset \neq \bar{O}_{\alpha j} \cap l \text { for some } i \neq j \text { implies } \\
B\left(a, \lambda r_{\alpha}\right) \cap l \subset\left(U_{\alpha} \sim \bigcup_{i=1}^{N} \bar{O}_{\alpha i}\right) \cap l
\end{gathered}
$$

for some $a \in l$.

Let $x \in M$ and let $V$ be the tangent plane of $M$ at $x$. Given $u, 1<u<2$, there are a neighborhood $W$ of $x$ in $R^{n}$ and a diffeomorphism $f$ of $\mathrm{W} \cap M$ into $V$ such that the Lipschitz constants $\operatorname{Lip}(f)$ and $\operatorname{Lip}\left(f^{-1}\right)$ are $\leqq u$. We denote

$$
A^{\prime}=f(A \cap W \cap M)
$$

for any $A \subset R^{n}$. Choosing $u$ sufficiently close to 1 we obtain from (1) for any line $l$ in $R^{n}$

$$
\begin{gathered}
\bar{O}_{\alpha i}^{\prime} \cap l \neq \emptyset \neq \bar{O}_{\alpha j}^{\prime} \cap l \quad \text { for some } i \neq j \text { implies } \\
B\left(a, \lambda r_{\alpha}\right) \cap l \subset\left(U_{\alpha}^{\prime} \sim \bigcup_{i=1}^{N} \bar{O}_{\alpha i}^{\prime}\right) \cap l
\end{gathered}
$$

for some $a \in l$ whenever $\bar{U}_{\alpha} \subset W$. 
Let $\varrho>0$ be such that $U$ contains a ball radius $\varrho$, let

$$
\varkappa=\left(8 / \varrho r_{1}\right)^{n} \text { and } \eta=\min \{1 /(4 \varkappa), \lambda /(8 \varkappa d(U))\},
$$

and let $t$ be the smallest positive number with the following property: If $I$ is any interval, $A \subset I$ and $I \sim A$ contains an interval of length $\eta d(I)$, then $A$ can be covered with two intervals $I_{1}, I_{2} \subset I$ such that $d\left(I_{1}\right)^{t}+d\left(I_{2}\right)^{t} \leqq d(I)^{t}$ and $\operatorname{dist}\left(I_{1}, I_{2}\right) \geqq \eta d(I)$. One of the intervals $I_{1}, I_{2}$ may be degenerate. Clearly $0<t<1$, and $t$ depends only on $\eta$.

Let $\alpha_{0}$ be such that $\bar{U}_{\alpha_{0}} \subset W$ and set

$$
H=f\left(K_{\alpha_{0}} \cap M\right) .
$$

Let $l$ be a line in $R^{n}$. We shall show that there exist closed intervals

$$
\begin{gathered}
I_{j_{1} \ldots j_{p}} \subset l, \quad j_{i}=1,2, i=1, \ldots, p, \quad p=1,2, \ldots, \quad \text { such that for all } p \\
H \cap l \subset \bigcup_{j_{1} \ldots j_{p}} I_{j_{1} \ldots j_{p}}, \\
I_{j_{1} \ldots j_{p} 1} \cup I_{j_{1} \ldots j_{p}} \subset I_{j_{1} \ldots j_{p}}, \\
d\left(I_{j_{1} \ldots j_{p} 1}\right)^{t}+d\left(I_{j_{1} \ldots j_{p} 2}\right)^{t} \leqq d\left(I_{j_{1} \ldots j_{p}}\right)^{t}, \\
\operatorname{dist}\left(I_{j_{1} \ldots j_{p} 1}, I_{j_{1} \ldots j_{p} 2}\right) \geqq \eta d\left(I_{j_{1} \ldots j_{p}}\right), \\
d\left(I_{j_{1} \ldots j_{p} j}\right) \leqq(1-\eta) d\left(I_{j_{1} \ldots j_{p}}\right), \quad j=1,2 .
\end{gathered}
$$

We first choose two closed intervals $I_{1}, I_{2}$ so that $H \cap l \subset I_{1} \cup I_{2}$ and $d\left(I_{j}\right) \leqq$ $d\left(U_{\alpha_{0}}\right)$ for $j=1,2$. Suppose then that $q \geqq 1$ and that $I_{j_{1} \ldots j_{p}}$ for $p=1, \ldots, q$ have been chosen so that (3)-(7) hold. Fix $I=I_{j_{1} \ldots j_{q}}$. We shall show that $I \sim H$ contains an interval of length $\eta d(I)$, whence the required intervals $I_{j_{1} \ldots j_{q} j}, j=1,2$, can be found by the choice of $t$.

Let $J \subset I$ be the interval in the middle of $I$ of length $d(I) / 2$. Let $A$ be the set of all those multi-indices $\alpha$ for which $\alpha_{0} \prec \alpha, \bar{O}_{\alpha} \cap J \neq \emptyset$,

$$
r_{1} d(I) / 8 \leqq d\left(U_{\alpha}\right)<d(I) / 8,
$$

and which are maximal (in the order $\prec$ ) with respect to these properties. Then $U_{\alpha}^{\prime} \cap l \subset I$ for $\alpha \in A$, because $\bar{U}_{\alpha}^{\prime} \cap J \neq \emptyset$ and $d\left(U_{\alpha}^{\prime}\right)<d(I) / 4$. For $\alpha \in A$ let $\alpha^{\prime}$ be the (unique) minimal sequence such that $\alpha \prec \alpha^{\prime}$ and $\bar{O}_{\alpha^{\prime} i}^{\prime} \cap \neq \emptyset \neq \bar{O}_{\alpha^{\prime} j}^{\prime} \cap l$ for some $i \neq j$, if such a sequence exists. We then set $P_{\alpha}=\bar{O}_{\alpha^{\prime}}^{\prime} \cap l$. If such a sequence does not exist, then either there are $\alpha \prec \alpha_{1} \prec \alpha_{2} \prec \ldots$ such that $\cap_{i=1}^{\infty} \bar{O}_{\alpha_{i}}^{\prime} \cap l$ is a singleton $\left\{x_{\alpha}\right\}$ or there is $\alpha^{\prime}$ such that $\alpha \prec \alpha^{\prime}, \bar{O}_{\alpha^{\prime}}^{\prime} \cap l \neq \emptyset$ and $\bar{O}_{\alpha^{\prime} i}^{\prime} \cap l=\emptyset$ for all $i$. In the first case we set $P_{\alpha}=\left\{x_{\alpha}\right\}$, in the second $P_{\alpha}=\emptyset$, and we agree $d(\emptyset)=0$. Then

$$
H \cap J \subset \bigcup_{\alpha \in A} P_{\alpha} .
$$

To see this let $x \in H \cap J$. Then there is an infinite sequence $\left(i_{1}, i_{2}, \ldots\right)$ such that $x \in \bar{O}_{\alpha_{p}}^{\prime} \cap l$ where $\alpha_{p}=\left(i_{1}, i_{2}, \ldots, i_{p}\right)$ for $p=1,2, \ldots$ and $\alpha_{0}=\alpha_{p_{0}}$ for some $p_{0}$. Since for $j=1$ or $2, d(I) \leqq d\left(I_{j}\right) \leqq d\left(U_{\alpha_{0}}\right)$, and since $r_{1} d\left(U_{\alpha_{p+1}}\right) \leqq r_{1} d\left(U_{\alpha_{p}}\right) \leqq d\left(U_{\alpha_{p+1}}\right)$, 
it follows from (8) that for some $p \geqq p_{0}, \alpha_{p} \in A$. Then one readily checks that $x \in P_{\alpha_{p}}$ which proves (9).

By the definition of $\varrho$, each $U_{\alpha}$ contairs a ball of radius $\varrho d\left(U_{\alpha}\right)$. Since the elements of $A$ are maximal, the sets $U_{\alpha}, \alpha \in A$, are disjoint. Moreover, they are contained in a ball of radius $d(I)$. If $k$ is the number of the elements of $A$, we see comparing volumes that $k\left(\varrho r_{1} d(I) / 8\right)^{n} \leqq d(I)^{n}$, that is, $k \leqq \varkappa$.

Let $\beta \in A$ be such that $d\left(P_{\beta}\right)$ is the largest of the diameters $d\left(P_{\alpha}\right), \alpha \in A$. If $2 \varkappa d\left(P_{\beta}\right)<d(J)$, then by (9) $I \sim H$ contains an interval of length $d(J) /(2 \varkappa) \geqq \eta d(I)$. If $2 \varkappa d\left(P_{\beta}\right) \geqq d(J)>0$, then $P_{\beta^{\prime}}=\bar{O}_{\beta^{\prime}}^{\prime} \cap l$ and $\bar{O}_{\beta^{\prime} i}^{\prime} \cap l \neq \emptyset \neq \bar{O}_{\beta^{\prime} j}^{\prime} \cap l$ for some $i \neq j$, and (2) implies that $\left(U_{\beta^{\prime}}^{\prime} \sim \bigcup_{i=1}^{N} \bar{O}_{\beta^{\prime} i}^{\prime}\right) \cap l$ contains an interval $I_{0}$ of length

$$
\lambda r_{\beta^{\prime}}=\lambda d\left(U_{\beta^{\prime}}\right) / d(U) \geqq \lambda d\left(P_{\beta}\right) /(2 d(U)) \geqq \lambda d(J) /(4 \varkappa d(U)) \geqq \eta d(I) .
$$

If $\beta^{\prime} \in C_{p}(N)$ then $U_{\beta^{\prime}}^{\prime} \cap \bar{U}_{\gamma}^{\prime}=\emptyset$ for $\gamma \in C_{p}(N), \gamma \neq \beta^{\prime}$; hence $H \cap U_{\beta^{\prime}} \subset \bigcup_{i=1}^{N} \bar{O}_{\beta^{\prime}}^{\prime}$, and it follows that $I_{0}$ is contained in $I \sim H$. This completes the induction.

From (5) we obtain for $p=1,2, \ldots$

$$
\sum_{j_{1} \ldots j_{p}} d\left(I_{j_{1} \ldots j_{p}}\right)^{t} \leqq d\left(I_{1}\right)^{t}+d\left(I_{2}\right)^{t}
$$

hence (3) and (7) imply

and $\operatorname{dim}(H \cap l) \leqq t$.

$$
\mathscr{H}^{t}(H \cap l) \leqq d\left(I_{1}\right)^{t}+d\left(I_{2}\right)^{t}<\infty,
$$

We set $\varepsilon=1-t$. Then $\operatorname{dim} H \leqq m-\varepsilon$. For otherwise it would follow from [5, Theorem 6.6] that for some lines $l, \operatorname{dim}(H \cap l)=\operatorname{dim} H+1-m>t$. Since $H$ is diffeomorphic to $K_{\alpha_{0}} \cap M$, we have $\operatorname{dim}\left(K_{\alpha_{0}} \cap M\right) \leqq m-\varepsilon$. This completes the proof.

3.2. Remarks. If the lines are replaced by $m$-planes in the assumptions of Theorem 3.1, then $\mathscr{H}^{m}(M \cap K)=0$ by [2,5.4(1)]. I do not know whether $\operatorname{dim}(M \cap K)<m$ in this case (except if $m=1$ ).

On the other hand Theorem 3.1 and also [2, 5.4 (1)] are false without some assumption in addition to the open set condition. For example, if $O$ is an open equilateral triangle in $R^{2}, O_{1}, O_{2}, O_{3} \subset O$ are homothetic to $O$ in ratio $1 / 2$ each having one vertex in common with $O$ and two sides contained in boundary $O$, and if $S_{1}, S_{2}, S_{3}$ are the obvious similitudes (without rotation) with $S_{i} O=O_{i}$, then $\mathscr{S}=\left\{S_{1}, S_{2}, S_{3}\right\}$ satisfies the open set condition with $O, \operatorname{dim} K=\log 3 / \log 2>1$, but $K$ contains countably many line segments. In this case the other separation condition fails for the lines containing the boundary segments of $O$. 


\section{The case $s \leqq m$}

Marstrand considered the concept weakly tangential in [4]. We give the following.

4.1. Definition. Let $E \subset R^{n}$ and $0 \leqq t \leqq n$. We say that $E$ is weakly $(t, m)$ tangential at a point $a \in R^{n}$ if $\Theta^{* t}(E, a)>0$ and there is an m-plane $V$ such that $a \in V$ and for every $\delta>0$

$$
\liminf _{r \downarrow 0} r^{-t} \mathscr{H}^{t}(E \cap B(a, r) \sim\{x: \operatorname{dist}(x, V) \leqq \delta r\})=0 .
$$

$V$ is then called a weak $(t, m)$ tangent plane of $E$ at a.

In general there may be several or no weak tangent planes. In the following recall that $K=|\mathscr{S}|, s=\operatorname{dim} K$ and $0<\mathscr{H}^{s}(K)<\infty$ due to the open set condition.

4.2. Theorem. Suppose that $\mathscr{S}$ satisfies the open set condition. If $K$ has a weak $(s, m)$ tangent plane $V$ at some point $a \in K$, then $K \subset V$.

Proof. We first show that $K$ lies on some $m$-plane. Suppose this is not true. Then there is a subset $\left\{a_{1}, \ldots, a_{m+2}\right\}$ of $K$ which is not contained in any $m$-plane, and we can find $\varrho, 0<\varrho<1$, such that if $W$ is an $m$-plane, then $\operatorname{dist}\left(a_{i}, W\right) \geqq \varrho$ for some $i=1, \ldots, m+2$. The lower densities $\Theta_{*}^{s}\left(K, a_{i}\right)$ are positive by $[2,5.3(1)]$. Hence there are $r_{0}, \eta$ such that $0<r_{0}<\varrho / 2$ and

$$
\mathscr{H}^{s}\left(K \cap B\left(a_{i}, r_{0}\right)\right) \geqq \eta r_{0}^{s} \quad \text { for } \quad i=1, \ldots, m+2 .
$$

Let $0<r<r_{1}$ and let $\alpha$ be a minimal sequence such that $a \in K_{\alpha} \subset B(a, r / 2)$. We may assume $d(K)=1$. Then $r_{\alpha} \leqq r / 2 \leqq r_{\alpha} / r_{1}$. By the choice of $\varrho$ there is $i$ such that $\operatorname{dist}\left(S_{\alpha}^{-1}(V), a_{i}\right) \geqq \varrho$. Then

$$
B\left(S_{\alpha} a_{i}, r_{\alpha} r_{0}\right) \subset B(a, r) \sim\left\{x: d(x, V) \leqq \varrho r_{\alpha} / 2\right\} .
$$

Hence with $\delta=\varrho r_{1} / 4$, we have $\delta r \leqq \varrho r_{\alpha} / 2$ and

$$
\begin{gathered}
\mathscr{H}^{s}(K \cap B(a, r) \sim\{x: d(x, V) \leqq \delta r\}) \geqq \mathscr{H}^{s}\left(K_{\alpha} \cap B\left(S_{\alpha} a_{i}, r_{\alpha} r_{0}\right)\right) \\
=r_{\alpha}^{s} \mathscr{H}^{s}\left(K \cap B\left(a_{i}, r_{0}\right)\right) \geqq \eta r_{\alpha}^{s} r_{0}^{s} \geqq \eta\left(r_{1} r_{0} / 2\right)^{s} r^{s} .
\end{gathered}
$$

This contradicts the fact that $V$ is a weak $(s, m)$ tangent plane for $K$ at $a$. Therefore $K \subset W$ for some $m$-plane $W$.

Suppose that $W \neq V$. Letting $X$ be the orthogonal complement of $V \cap W$, we have

$$
c=\operatorname{dist}(W \cap X \cap\{x:|x|=1\}, V \cap X)>0 .
$$

Then one checks $c$ dist $(x, V \cap W) \leqq \operatorname{dist}(x, V)$ for $x \in W$. Since $K \subset W$ and $\Theta_{s}^{*}(K, a)>0$, it follows that $1 \leqq \operatorname{dim}(V \cap W)<m$ and that $V \cap W$ is a weak tangent plane for $K$ at $a$.

Let $k, 0<k<m$, be the smallest integer such that $K$ is weakly $(s, k)$ tangential at $a$. Then by the above proof $K$ lies on some $k$-plane $U \subset W$. Then $U \subset V$, because 
otherwise $U \cap V$ would again be a weak tangent plane for $K$ at $a$ with $1 \leqq \operatorname{dim}$ $(U \cap V)<k$, which would contradict the choice of $k$. Thus $K \subset V$.

4.3. Corollary. Either $K$ lies on an m-plane or $\mathscr{H}^{s}(M \cap K)=0$ for every $m$ dimensional $C^{1}$ submanifold $M$ of $R^{n}$.

Proof. Suppose $\mathscr{H}^{s}(M \cap K)>0$ for some $M$. For $\mathscr{H}^{s}$ a.a. $x \in M \cap K$, $\Theta^{* s}(K \sim M, x)=0$ by [1,2.10.19 (4)], and at these points the tangent $m$-plane of $M$ is a weak $(s, m)$ tangent plane of $K$. Hence $K$ lies on an $m$-plane.

4.4. Corollary. If either $s$ is non-integral or $s$ is an integer and $K$ lies on no s-plane, then

$$
\Theta_{*}^{s}(K, x)<\Theta^{* s}(K, x) \text { for } \mathscr{H}^{s} \text { a.a. } x \in K \text {. }
$$

Proof. If $s$ is non-integral this follows directly from Marstrand's result [4]. If $s$ is an integer set $E=\left\{x \in K: \Theta_{s}^{*}(K, x)=\Theta^{* s}(K, x)\right\}$, then by [4] $K$ is weakly $(s, s)$ tangential for $\mathscr{H}^{s}$ a.a. $x \in E$. Hence by Theorem $4.2, \mathscr{H}^{s}(E)=0$.

4.5. Remark. If $s$ is an integer and $K$ lies on no $s$-plane then Theorem 4.2 implies that $K$ is purely $\left(\mathscr{H}^{s}, s\right)$ unrectifiable in the sense of [1, 3.2.14]. It is not known whether the conclusion of 4.4 is true for all purely $\left(\mathscr{H}^{s}, s\right)$ unrectifiable sets $K \subset R^{n}$ with $\mathscr{H}^{s}(K)<\infty$, except if $s=1$.

\section{References}

[1] Federer, H.: Geometric measure theory. - Die Grundlehren der mathematischen Wissenschaften 153, Springer-Verlag, Berlin-Heidelberg-New York. 1969.

[2] Hutchinson, J. E.: Fractals and self-similarity. - Indiana Univ. Math. J. 30, 1981, 713747.

[3] Mandelbrot, B. B.: Fractals: form, chance, and dimension. - W. H. Freeman and Company, San Francisco, 1977.

[4] Marstrand, J. M.: The $(\varphi, s)$ regular subsets of $n$ space. - Trans. Amer. Math. Soc. 113, 1964, 369-392.

[5] Mattila, P.: Hausdorff dimension, orthogonal projections and intersections with planes. Ann. Acad. Sci. Fenn. Ser. A I Math., 1975, 227-244.

The Institute for Advanced Study

School of Mathematics

Princeton, New Jersey 08540

USA

Received 6 November 1981
University of Helsinki

Department of Mathematics

SF-0010 Helsinki 10

Finland 